\title{
Closed reduction and percutaneous annulated screw fixation in the treatment of comminuted proximal humeral fractures
}

\author{
Zhe $\mathrm{Yu}^{\mathrm{D}}$, Lianhe Zheng ${ }^{\mathrm{B}}$, Xiaodong Yan ${ }^{\mathrm{C}}$, Xiaoxiang Li', Jian Zhao ${ }^{\mathrm{B}}$, Bao'an $\mathrm{Ma}^{\mathrm{A}}$ \\ Department of Orthopedic Surgery, Tangdu Hospital, Fourth Military Medical University, Xi'an, Shaanxi, China \\ A - research concept and design; B - collection and/or assembly of data; $\mathrm{C}$ - data analysis and interpretation; \\ $D$ - writing the article; $E$ - critical revision of the article; $F$ - final approval of article
}

\section{Address for correspondence \\ Zhe Yu \\ E-mail: 48470941@qq.com}

Funding sources

This study was supported by the National Natural Science foundation (31670940/C080904, CHN), the Chinese Postdoctoral Science Foundation funded project (grant no. 2013M542441 and 2012T50825, (HN) and the Natural Science Foundation research project of Shaanxi Province (S2016YFJM0737).

\section{Conflict of interest}

none declared

\section{Acknowledgments}

The authors thank Dr. Jiachang Wu and Dr. Tongshuan Gao for assistance with data processing.

Received on March 4, 2014

Revised on April 28, 2014

Accepted on September 23, 2014

\begin{abstract}
Background. Displaced proximal humeral fractures remain a challenge to orthopedic surgeons.

Objectives. The purpose of this study was to evaluate the functional and radiological outcomes of patients with comminuted proximal humeral fractures treated with closed reduction and percutaneous screw fixation (CRPF).

Material and methods. The authors retrospectively reviewed 38 cases of displaced proximal humeral fractures (2-, 3- or 4-part fractures according to the Neer classification) that were treated using the CRPF technique from May 2009 to April 2013. From this group 26 patients were followed up for a period ranging from 9 to 24 months (averaging 12.9 months) and evaluated for the functional and radiological outcomes by a series of standard questionnaires and measurements.
\end{abstract}

Results. The fractures in all 26 patients were healed within an average time of 14.6 weeks (ranging from 11 to 27 weeks), and the mean interval between the operation and fully functional activity was 18.6 weeks (ranging from 15 to 32 weeks). At the final follow-up visit, no patient showed shoulder instability; the mean range of abduction motion was $146.5^{\circ}$ (ranging from $72^{\circ}$ to $180^{\circ}$ ). For all patients, no statistically significant difference in the functional outcomes was observed between their 6-month and final follow-up visits; or in the radiological findings between their immediate post-operative and final follow-up examinations.

Conclusions. The CRPF technique is a safe and effective therapeutic option for comminuted proximal humeral fractures. Good stability is obtained and aggressive impairment of the soft tissue and periosteum around the fracture is avoided, which allows for an early painless range of motion. The technique promotes bone healing, prevents ischemic osteonecrosis of the head of the humerus and leads to few complications.

Key words: functional outcome, radiological outcome, proximal humeral fracture, fracture fixation, percutaneous technique

DOI

10.17219/acem/28898

Copyright

Copyright by Author(s)

This is an article distributed under the terms of the

Creative Commons Attribution Non-Commercial License

(http://creativecommons.org/licenses/by-nc-nd/4.0/) 
Although comminuted proximal humeral fractures are among the most common fractures in the elderly population, treatment of this injury remains a challenge and is still an issue of debate. The Neer classification is one of the most popular systems for assessing fractures of this kind. No displaced or minimally displaced fractures can be treated conservatively with success. Displaced 2-part, 3-part and 4-part fractures should be treated for reduction and stabilization.

Considerable therapeutic options for these fractures have been described in past years. ${ }^{1}$ However, for some elderly osteoporosis patients or more comminuted cases, the stability of percutaneous pinning fixation cannot meet the requirements of fixation and early functional activity. Therefore, compressing inter-fracture fragments is of great importance for restoring post-operative motor function.

Percutaneous annulated screw fixation, first described by Chen et al., was developed specifically for comminuted proximal humeral fractures, and appeared to offer improved fixation intensity of these fractures through the use of multiple annulated compression screws oriented in different directions to maximize the screw compression effects and the resistance to displacement. ${ }^{2}$ Although this technique is demanding, it can be very effective for unstable 2-part and 3-part fractures and even some 4-part fractures in patients whose bone quality is not good.

The aim of this study was to evaluate the post-operative outcomes of 26 patients with displaced proximal humeral fractures treated with closed reduction and percutaneous screw fixation (CRPF). The authors reviewed the functional outcomes, radiographic outcomes and complications to investigate the hypothesis that CRPF is an effective therapeutic alternative for unstable proximal humeral fractures.

This was a retrospective clinical study and was approved by the Institutional Review Board of the Fourth Military Medical University and Tangdu Hospital (Xi'an, China). All the participants provided their written informed consent to participate in this study, and the ethics committee approved the consent procedure.

\section{Material and methods}

\section{Patients}

From May 2009 to April 2013, 38 patients with closed displaced proximal humeral fractures were treated using the CRPF technique at the Department of Orthopedic Surgery, Tangdu Hospital (Xi'an, China). Open reduction and internal fixation (ORIF) treatment was performed when the initial closed reduction failed or the fracture had re-displaced on repeat radiographs taken 5-7 days later. All fractures were stabilized with the percutaneous annulated screw fixation technique by experienced orthopedic surgeons. Out of the 38 patients, 26 were evalu- ated for their clinical outcomes, and the other 12 patients were excluded: 2 patients changed their address or phone number and could not be contacted, 3 declined participation, 5 suffered closed reduction failure or the fracture had re-displaced and underwent ORIF treatment, and the remaining 2 patients suffered humeral head splitting fractures involving the articular surface, which were not suitable for CRPF treatment. Open fracture injuries were not fit for this therapeutic technique, and were not brought into this study. The 26 participants' general information and fracture types were recorded preoperatively (Table 1).

Table 1. General patient information and fracture types

\begin{tabular}{|lc|}
\hline Details & Number (\%) \\
\hline Age (years) & 63.6 (range: $36-79)$ \\
$<60$ years old & $9(34.6)$ \\
$>60$ years old & $17(65.4)$ \\
Side & left: $11(42.3)$ \\
& right $15(57.7)$ \\
Gender & female $8(30.8)$ \\
& male $18(69.2)$ \\
Isolated fracture & $20(76.9)$ \\
Multiple fractures & $6(23.1)$ \\
Fracture pattern & 2-part $7(26.9)$ \\
(Neer classification) & 3-part 17 (65.4) \\
& 4 -part 2(7.7) \\
\hline
\end{tabular}

\section{Operative planning and surgical technique}

The authors' CRPF technique has been described in detail elsewhere but will be reviewed in brief here. ${ }^{3}$ All the patients were treated surgically within 2 weeks of the injury (Fig. 1a). The operations were carried out with the patient in a modified beach-chair or supine position with the involved scapula positioned over the edge of the table. Under general anesthesia or nerve-blocking anesthesia, with fluoroscopic control, closed reduction was realized to obtain good fragment contact and alignment. Guide pins were inserted through the deltoid into the humerus towards the humeral head, or crossing from the greater tubercle to the distal fracture end.

Good fragment alignment and the position of the guide pins were confirmed by fluoroscopic imaging. Then 4.5-mm AO/ASIF annulated compression screws were used for fixation along the guide pins. Three to 4 screws were inserted in each case (Fig. 1b). After the insertion of the screws for compression fixation, all of the guide pins were extracted, and each incision was closed with number-5 Ethicon sutures.

Post-operatively, the shoulder was passively moved with gradually improved range. In severely comminuted cases, the shoulder was fixed in a shoulder brace for 3-4 weeks, and then shoulder rehabilitation was performed gradually. The patients were examined in the outpatient clinic 


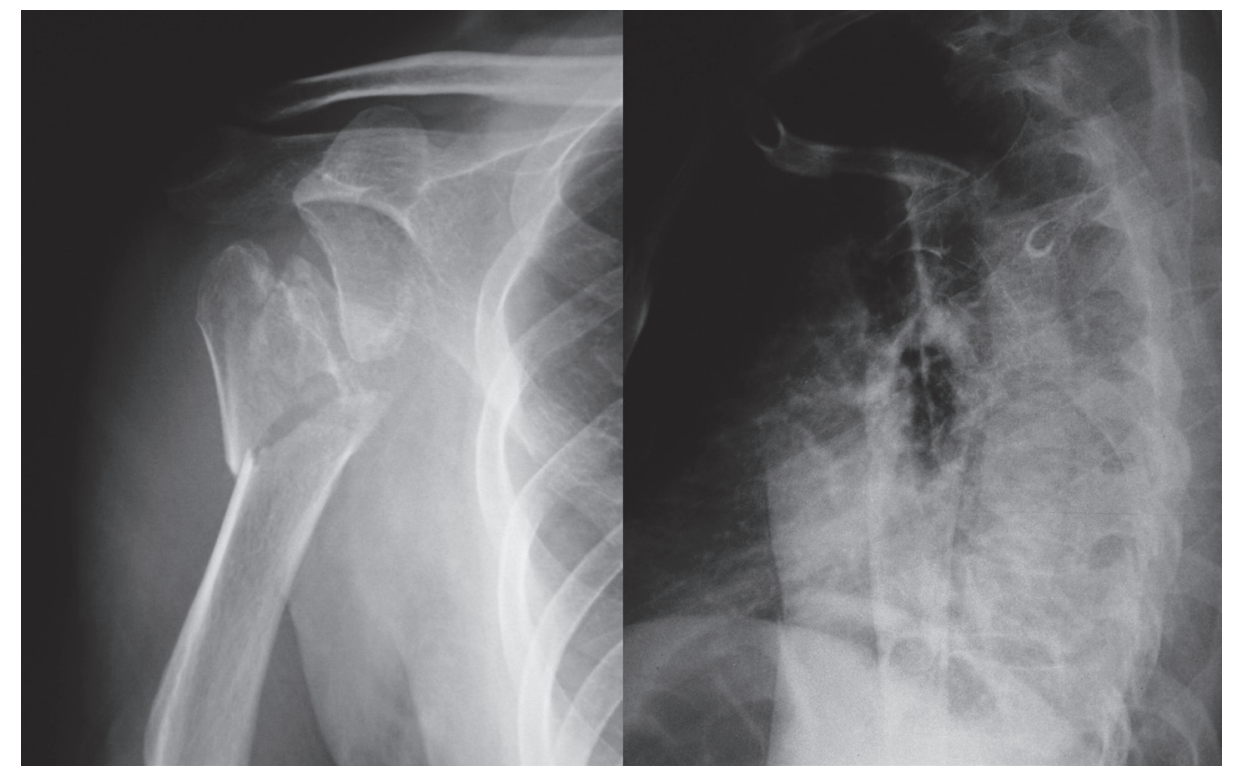

Fig. 1a. Anteroposterior and lateral position radiographs showing a displaced 3-part fracture of the proximal humerus involving the surgical neck and greater tuberosity

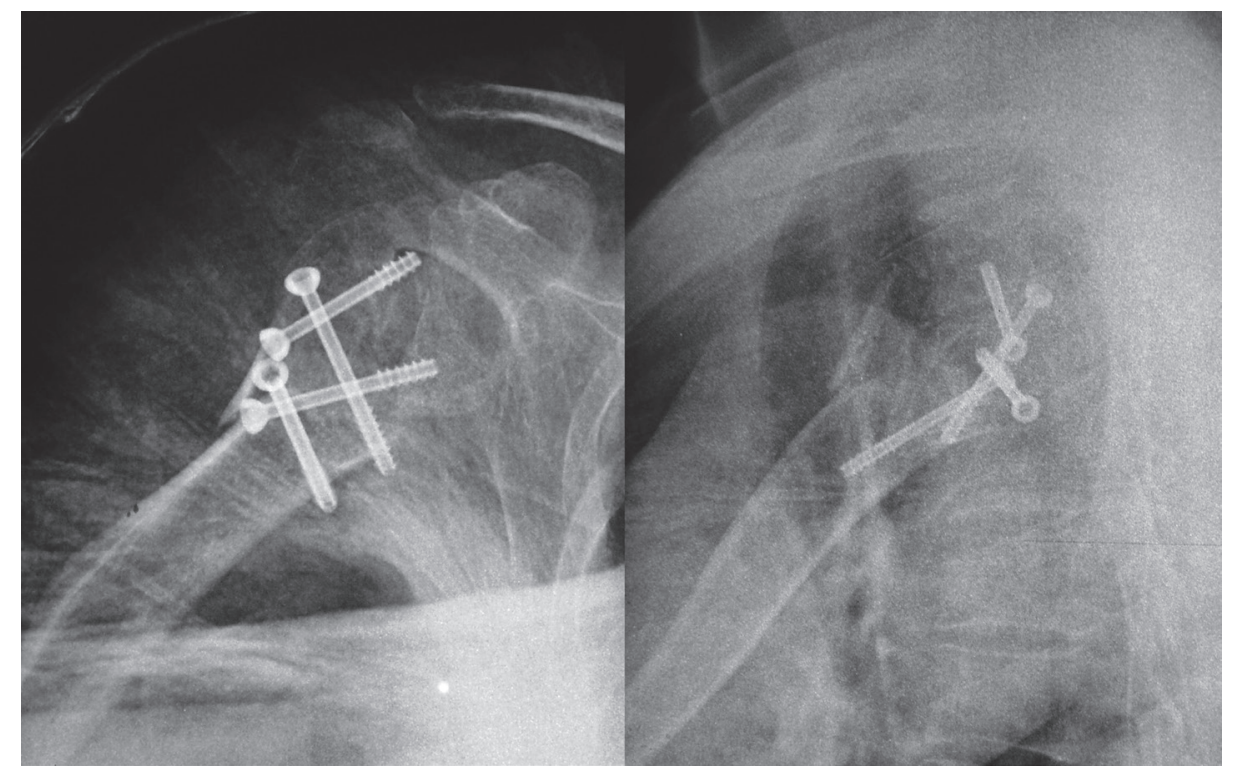

Fig. 1b. Anteroposterior and lateral position radiographs taken immediately after the operation, showing the head and greater tuberosity fragment with alignment restored and fixed with 4 annulated compression screws oriented in different directions

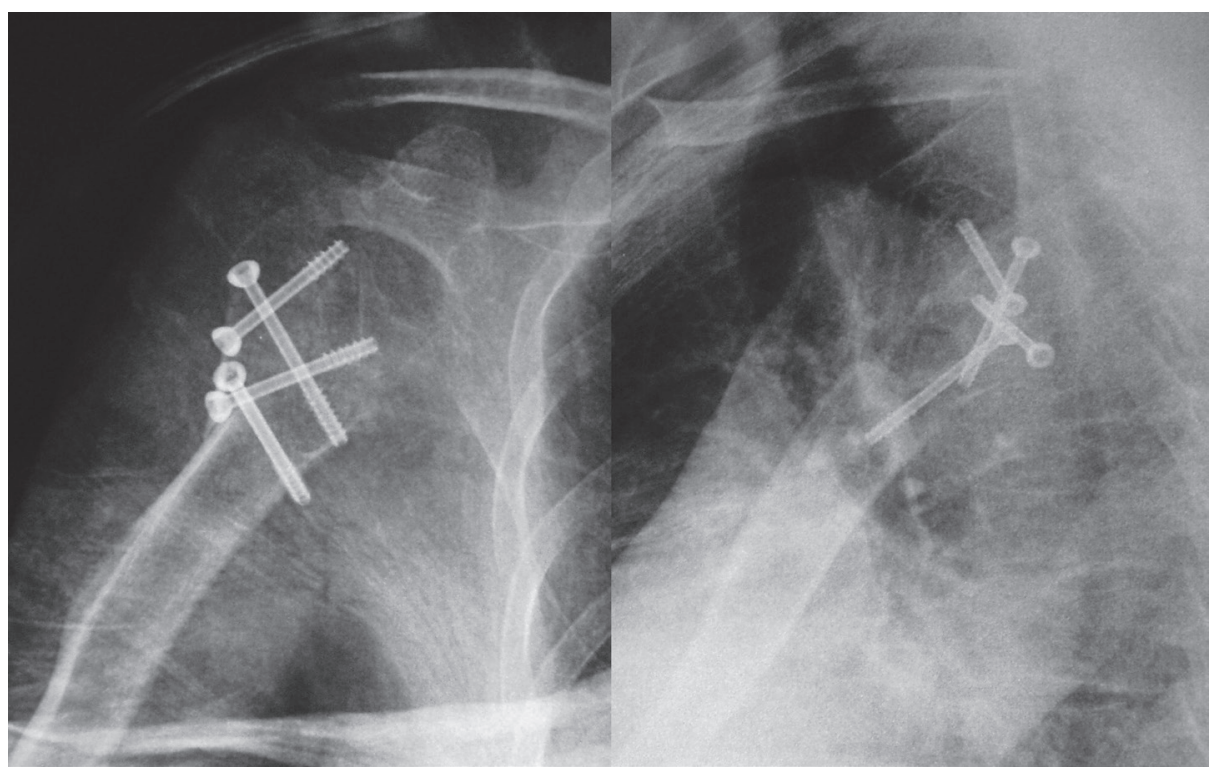

Fig. 1c. AP and lateral position radiographs taken 3 months after the operation, showing the bridging bone between the fragments, which both indicate that bone union has been achieved 
3 weeks, 6 weeks, 3 months, and 1-2 years after surgery. The AP position and lateral position were obtained through chest X-rays at each visit to assess alignment, union and any signs of avascular necrosis (Fig. 1c).

\section{Follow-up}

A total of 26 patients participated in the follow-up, which ranged from 9 to 24 months (averaging 12.9 months). The patients Constant scores and American Shoulder and Elbow Surgeons (ASES) scores, along with a visual analog scale (VAS) survey, were registered at the 6-month postoperative follow-up and the subsequent follow-up visits when the patients attended an outpatient clinic.

Chest radiographs to check the post-operative AP and lateral position of the shoulder were taken at week 6 , week 12 and every subsequent $6^{\text {th }}$ week until bony union was achieved. Bone healing was determined by a combination of painless palpation of the shoulder and radiographic evidence of bridging bone on AP and lateral radiographs.

\section{Radiographic studies}

All the patients enrolled in the study had accessible, immediate post-operative radiographs. Radiological bony union was determined by the attending surgeon as at least 3 cortical unions. To score the residual deformity, angulation and displacement were considered independently, and each fragment (the humeral head, greater tuberosity and lesser tuberosity) was scored individually. The final score for each case was the sum of the scores allocated to each fragment. An angulation between $20^{\circ}$ and $45^{\circ}$ was scored as 1 point and $>45^{\circ}$ was scored as 2 points. A displacement between 0.5 and $1 \mathrm{~cm}$ was scored as 1 point and $>1 \mathrm{~cm}$ was scored as 2 points. If the angulation and displacement were lower than $20^{\circ}$ and $0.5 \mathrm{~cm}$, respectively, the quality of the reduction was considered excellent and scored as 0 points. ${ }^{4}$ Data are expressed as mean \pm standard deviation (SD).

\section{Clinical evaluation}

Clinical evaluation of the results was done in accordance with the patients' VAS surveys, Constant scores and ASES scores a minimum of 1 year post-operatively.

Pain was assessed with the VAS, with a maximum of 10 points for this evaluation. Physical examination included measurements of the passive and active ranges of motion of the shoulder with a standard goniometer. Care was taken to prevent compensatory trunk movement during the shoulder range-of-motion measurements.

The Constant score is a 100-point scoring system composed of the following parameters: Pain, daily life activities, range of motion and power. Only the active range of motion was considered. The range of motion was measured and compared with the opposite shoulder.
The ASES questionnaire, which was designed by the research committee of the American Shoulder and Elbow Surgeons in 1994, score totals 100 points and allocates 50 points for measuring function and 50 points for pain. The goal of this questionnaire was to develop a standardized method for evaluating shoulder function based entirely on patient self-evaluation.

\section{Statistical analysis}

SPSS software (v. 11.0, SPSS Inc., Chicago, USA) was used for the data analysis. Statistical comparisons of the patients' 6-month post-operative functional scores and the final follow-up were done using Student's t-test, with statistical significance set at $\mathrm{p}<0.05$. Means were compared by use of the Kruskal-Wallis test if the data did not follow a normal distribution. Similarly, comparisons of residual deformity in the post-operative radiographs and the final follow-up were also carried out using Student's t-test.

\section{Results}

As shown in Table 2, all the fractures were healed after a follow-up ranging from 9 to 24 months (averaging 12.9 months). There were no superficial or deep wound infections, nor were there any nerve palsies after surgery. There was 1 case of varus abnormality, 2 cases of stiffness of the shoulder joint, 2 cases of ischemic osteonecrosis, 1 case of delayed union, 2 cases suffering from migration of at least one screw (screw removal surgery was performed about 6 months post-operatively, and fortunately no migration of fracture fragments was seen), and 4 cases of traumatic arthritis of shoulder joint.

Table 2. Surgical details of the 26 cases of comminuted proximal humeral fractures

\begin{tabular}{|c|c|}
\hline Details & Number (\%) \\
\hline Average time to surgery & 6.8 days (ranging 0-16 days) \\
\hline Pre-operative preparation & $\begin{array}{l}\text { traction: } 5 \text { (19.2) } \\
\text { external fixator or brace: } 9 \text { (34.6) } \\
\text { emergency operation: } 12 \text { (46.2) }\end{array}$ \\
\hline Mean operation time & 48.5 min (ranging 24-96 min) \\
\hline Number of implants & $\begin{array}{l}2: 3(11.5) \\
3: 13(50.0) \\
4: 10(38.5)\end{array}$ \\
\hline $\begin{array}{l}\text { Post-operative immobilized } \\
\text { time }\end{array}$ & $\begin{array}{l}\text { under } 1 \text { week: } 5 \text { (19.2) } \\
\text { 1-2 weeks: } 10 \text { (38.5) } \\
\text { 2-3 weeks: } 6(23.1) \\
\text { 3-6 weeks: } 3 \text { (11.5) } \\
6 \text { weeks or more: } 2 \text { (7.7) }\end{array}$ \\
\hline Main complication & $\begin{array}{l}\text { varus abnormality: } 1 \text { (3.8) } \\
\text { joint stiffness: } 2 \text { (7.7) } \\
\text { ischemic osteonecrosis: } 2 \text { (7.7) } \\
\text { traumatic arthritis: } 4 \text { (15.4) } \\
\text { delayed union: } 1 \text { (3.8) } \\
\text { migration of screws: } 2 \text { (7.7) }\end{array}$ \\
\hline
\end{tabular}




\section{Clinical evaluations of post-operative follow-up}

All fractures healed within a post-operative followup period ranging from 9 to 24 months (averaging 12.9 months). The mean interval between the operation and bone union was 14.6 weeks (ranging from 11 to 27 weeks), and the mean time between the operation and fully functional activity was 18.6 weeks (ranging from 15 to 32 weeks). At the final clinical evaluation, shoulder stability was achieved in all the patients, and the mean range of abduction motion was $146.5^{\circ}\left(72^{\circ}-180^{\circ}\right)$ (Table 3$)$.

Table 3. Clinical data of the 26 cases of comminuted proximal humeral fractures

\begin{tabular}{|c|c|}
\hline Details & Number \\
\hline Mean follow-up period & 12.9 months (range: $9-24$ months) \\
\hline Mean range of abduction motion & $146.5^{\circ}$ (range: $\left.72^{\circ}-180^{\circ}\right)$ \\
\hline Mean time of bone union & 14.6 weeks (range: 11-27 weeks) \\
\hline $\begin{array}{l}\text { Mean time to fully functional } \\
\text { activity }\end{array}$ & 18.6 weeks (range: 15-32 weeks) \\
\hline
\end{tabular}

\section{Radiological evaluations}

All cases except for 1 delayed union achieved radiographic union within 3 months. Table 4 shows the degree of residual deformity along with the fracture type according to the Neer classification. No significant differences were observed between the immediate post-operative imaging and the final follow-up imaging.

The statistical analysis demonstrated that the number of fragments displaced in the fracture showed a trend toward correlating with the residual deformity (i.e., the higher the number of fragments, the higher the residual deformity, $\mathrm{p}<0.05$ ). This means that the higher the severity of the fracture, the worse the quality of reduction and the higher the residual deformity after surgical treatment.

\section{Post-operative functional evaluations}

At the 6-month follow-up, the VAS pain score, the Constant score and the ASES score were respectively $1.72 \pm 1.24,73.6 \pm 24.8$ and $77.4 \pm 20.5$. At the final follow-up, these 3 scores were respectively $1.62 \pm 0.97$, $79.5 \pm 24.7$ and $80.6 \pm 15.3$. As shown in Table 5, there was no significant difference between the 6-month postoperative score and the final follow-up score in any of the 3 scoring systems.

The VAS was used to measure the average daily pain experienced in the shoulder for each patient. For the purposes of the statistical analysis, the patients were divided into 3 groups based on the Neer classification: 2-part, 3-part and 4-part fractures. The mean VAS pain score for each group was 1.46 for 2-part fractures, 1.78 for 3-part fractures, and 1.95 for 4-part fractures. There was no significant difference in this functional evaluation between the 3 fracture types $(p>0.05)$, or between the 6 -month visit and the final follow-up ( $>0.05)$.

The mean Constant score for the entire group was 73.6. At the 6-month follow-up visit the mean Constant score for the 3 fractures types was 75.5 for 2-part fractures, 73.4 for 3-part fractures and 59.2 for 4-part fractures. There was no significant difference in the mean Constant score for the 3 fracture types between the 6-month follow-up visit and the final follow-up ( $p>0.05)$, but it decreased

Table 4. Radiological outcomes of the patients

\begin{tabular}{|c|c|c|c|}
\hline Fracture classification & Post-operative & Final follow-up & $p$-value \\
\hline 2-part fracture (7 cases) & $1.7 \pm 1.2$ & $1.5 \pm 1.1$ & $>0.05$ \\
\hline 3-part fracture (17 cases) & $2.3 \pm 1.9$ & $2.2 \pm 1.8$ & $>0.05$ \\
\hline 4-part fracture (2 cases) & $3.9 \pm 0.7$ & $3.8 \pm 0.7$ & $>0.05$ \\
\hline Mean value (26 cases) & $2.0 \pm 1.5$ & $1.9 \pm 1.3$ & $>0.05$ \\
\hline
\end{tabular}

Table 5. Post-operative functional scores of the patients

\begin{tabular}{|c|c|c|c|c|}
\hline Fracture classification & & $\begin{array}{c}\text { Post-operative } \\
\text { (6 months) }\end{array}$ & Final follow-up & $p$-value \\
\hline VAS & $\begin{array}{l}\text { 2-part fracture } \\
\text { 3-part fracture } \\
\text { 4-part fracture }\end{array}$ & $\begin{array}{l}1.46 \pm 1.13 \\
1.78 \pm 0.94 \\
1.95 \pm 0.38\end{array}$ & $\begin{array}{l}1.51 \pm 1.22 \\
1.63 \pm 1.06 \\
1.75 \pm 0.28\end{array}$ & $>0.05$ \\
\hline Constant score & $\begin{array}{l}\text { 2-part fracture } \\
\text { 3-part fracture } \\
\text { 4-part fracture }\end{array}$ & $\begin{array}{l}75.5 \pm 25.8 \\
73.4 \pm 23.5 \\
59.2 \pm 12.4\end{array}$ & $\begin{array}{l}79.5 \pm 23.1 \\
74.9 \pm 18.4 \\
60.5 \pm 7.21\end{array}$ & $>0.05$ \\
\hline ASES score & $\begin{array}{l}\text { 2-part fracture } \\
\text { 3-part fracture } \\
\text { 4-part fracture }\end{array}$ & $\begin{array}{l}79.9 \pm 17.3 \\
76.2 \pm 24.2 \\
66.8 \pm 10.5\end{array}$ & $\begin{array}{c}82.5 \pm 12.7 \\
79.0 \pm 17.5 \\
68.4 \pm 9.3\end{array}$ & $>0.05$ \\
\hline
\end{tabular}


from 79.5 to $60.5(\mathrm{p}<0.05)$ with the increase of fracture fragment numbers.

The ASES score totals 100 points and allocates 50 points for measuring function and 50 points for pain. The format for this questionnaire has several advantages: It is relatively quick to complete, it is simple to score, it can be administered by telephone or by mail/internet, and it is widely accepted by most researchers. ${ }^{5}$ In this study, the mean ASES score was also calculated for the aforementioned 3 fracture types; it was 79.9 for 2-part fractures, 76.2 for 3-part fractures and 66.8 for 4-part fractures. The mean ASES score improved from 77.4 to $80.6(\mathrm{p}>0.05)$ between the 6-month follow-up and the final evaluation.

\section{Discussion}

Displaced proximal humeral fractures remain a challenge to orthopedic surgeons, usually resulting in malunion and ischemic osteonecrosis from conservative management or poor shoulder function due to aggressive impairment. In conservative treatment, using an external brace often causes permanent joint stiffness because of the restriction of early functional motion. At the same time, traditional ORIF with insertion of a buttress plate through a curve incision usually requires extensive stripping of the complicated soft tissue, including the pectoralis major insertion, caput longum musculi bicipitis brachii, and even the subscapularis muscle, which can often lead to devascularization of fracture fragments, permanent joint stiffness and increased risks of infection and nonunion.

Recently, the CRPF technique has gained popularity due to improved rigidness, compared with traditional conservative treatment or closed reduction and percutaneous pinning (CRPP) fixation. The compression of fracture fragments from the screw thread provides better fixation intensity, which consequently improves the healing rate, shortens the healing time and decreases ischemic osteonecrosis of the head of the humerus. Based on the results of this paper, for nearly $60 \%$ of the patients post-operative immobilized time is no more than 2 weeks, and for more than $80 \%$ percent of the patients immobilized time is 3 weeks or under. The main purpose of immobilization is to eliminate post-operative swelling of soft tissue, promote muscle healing and to provide sufficient time for the formation of primary bone callus between the fracture fragments, all of which are requisite conditions for achieving fully functional activity post-operatively. The main treatment goals in proximal humeral fractures are to restore the joint stability, congruity and alignment with minimal soft tissue dissection to allow early joint motion and good shoulder function. As a typical minimally invasive surgical technique, CRPF shows considerable advantages in minimizing soft tissue injury and enabling the patients to perform functional activities much earlier.
Soft tissue preservation is also a very critical issue in the treatment of comminuted proximal humeral fractures, because such a surgical approach with inadequate exposure often involves some important soft tissue injury, such as the axillary nerve, radial nerve, axillary vessels and rotator cuff. ${ }^{6-8}$ When percutaneous techniques are used, knowledge of anatomy is very important. Otherwise, even when percutaneous guide pins are inserted, the adjacent neurovascular bundle is susceptible to injury by the guide pins. The guide pins on the lateral cortex should be placed in a safe zone that avoids both the radial and axillary nerves. The radial nerve is relatively safe if the guide pins are kept above the deltoid insertion. The axillary nerve is located an average of $5 \mathrm{~cm}$ distal to the acromion; however, it may take a more variable path, particularly the anterior branches. ${ }^{9}$ When placing screws through the deltoid, a protective sleeve should be used to decrease the risk of nerve injury. It is also important to ensure that humeral retroversion averages $19^{\circ}$, and percutaneous guide pins or screws must be directed posteromedially to achieve this angle.

In the authors' experience, CRPP is rather a demanding surgical technique. First, it is not very easy to achieve acceptable reduction using indirect reduction techniques. If acceptable alignment cannot be achieved, the technique should be abandoned in favor of a more traditional open reduction. ${ }^{10}$ Poor bone quality and fracture comminuting are relative contraindications to this technique. Also, the patients must be cooperative and able to comply with the post-operative protocol and rehabilitation. Therefore, careful patient selection is recommended, as well as close follow-up in the first 4 weeks after surgery to minimize loss of reduction and fixation.

This study also focused on possible operative risks and post-operative complications. The $1^{\text {st }}$ risk is that CRPF is truly technically demanding, and during the operation process, surgeons are exposed to radiation, and may need to try more than once to select very suitable annulated lag screws after the closed reduction and temporary guide pin fixation have been achieved. Of course, the process of placing screws should also be done very carefully, since improper fixing of fragile fragments can lead to further comminuting. Removal of the screws after facture healing is achieved is also quite a difficult process because the percutaneous incisions are usually small and the deltoid muscle is rather strong. The removal operation often requires a longer incision than the therapeutic process; just as everything in the world has 2 sides, a minimally invasive surgical technique will inevitably entail more difficulties during internal fixation removal.

\section{References}

1. Carbone S, Tangari M, Gumina S, Postacchini R, Campi A, Postacchini F. Percutaneous pinning of three- or four-part fractures of the proximal humerus in elderly patients in poor general condition: MIROS $^{\circledR}$ versus traditional pinning. Int Orthop. 2012;36(6):1267-1273. 
2. Chen $\mathrm{CY}$, Chao EK, Tu YK, Ueng SW, Shih $\mathrm{CH}$. Closed management and percutaneous fixation of unstable proximal humerus fractures. J Trauma. 1998;45(6):1039-1045.

3. Keener JD, Parsons BO, Flatow EL, Rogers K, Williams GR, Galatz LM Outcomes after percutaneous reduction and fixation of proximal humeral fractures. J Shoulder Elbow Surg. 2007;16(3):330-338.

4. Harrison AK, Gruson KI, Zmistowski B, et al. Intermediate outcomes following percutaneous fixation of proximal humeral fractures. J Bone Joint Surg Am. 2012;94(13):1223-1228.

5. Sallay PI, Reed L. The measurement of normative American Shoulder and Elbow Surgeons scores. J Shoulder Elbow Surg. 2003;12(6): 622-627.
6. Cheung S, Fitzpatrick M, Lee TQ. Effects of shoulder position on axillary nerve positions during the split lateral deltoid approach. J Shoulder Elbow Surg. 2009;18(5):748-755.

7. Rancan $M$, Dietrich $M$, Lamdark T, Can U, Platz A. Minimal invasive long PHILOS ${ }^{\circledR}$-plate osteosynthesis in metadiaphyseal fractures of the proximal humerus. Injury. 2010;41(12):1277-1283.

8. Watson M. Major ruptures of the rotator cuff. The results of surgical repair in 89 patients. J Bone Joint Surg Br. 1985;67(4):618-624.

9. Sung CM, Roh GS, Sohn HJ, Park HB. Prediction of the location of the anterior branch of the axillary nerve, using correlations with physical factors: a cadaveric study. J Shoulder Elbow Surg. 2013;22(11):e9-e16.

10. Röderer G, Erhardt J, Graf M, Kinzl L, Gebhard F. Clinical results for minimally invasive locked plating of proximal humerus fractures. J Orthop Trauma. 2010;24(7):400-406. 